\title{
Los maestros y su función como fomentadores del valor de la solidaridad en el proceso de enseñanza - aprendizaje
}

\section{Teachers and their work as promoters of the solidarity value on the process teaching learning}

\section{Dr. Carlos Adolfo Sanabria Valdés ${ }^{1}$.}

Universidad Autónoma Metropolitana, Unidad

Azcapotzalco, México.

\section{RESUMEN}

Los maestros son agentes de cambio que pueden fomentar la solidaridad entre los estudiantes. Esto se lleva a cabo mediante la implementación de la Escuela Crítica. Una característica de esta última es la construcción del conocimiento en equipos de trabajo. En este último modelo pedagógico, los estudiantes trabajan en equipos dentro y fuera del salón de clases. Cuando se lleva a cabo lo anterior, los partamento de derecho de la Universidad Autónoma Metropolitana, Unidad Azcapotzalco, México.

ORCIDiD 0000-0002-8055-3267

Google académico: carlossanabriavaldes@gmail.com integrantes del equipo se retroalimentan para entregar un trabajo solicitado por el maestro. El trabajo en equipo de alumnos fomenta la colaboración y la solidaridad de cada persona del equipo. La solidaridad fomentada por el docente cuando aplica cualquier técnica grupal, produce una especie de ayuda entre los estudiantes. La ayuda es una parte de la solidaridad del ser humano. La primera es un valor que permite reconocer la vida de otro ser humano y su dignidad como persona. El docente es un agente de cambio para fomentar la solidaridad a través del proceso de enseñanza aprendizaje, en especial cuando éste aplica el trabajo en equipo en el aula. 


\section{ABSTRACT}

Teachers are agents of change who can foster solidarity among students. This last idea is according to applying Critical School. One characteristic of this School is building knowledge in groups of working. In this last pedagogical model, students work in teams inside and outside of the classroom. When this kind of working is done, students of the team feed each other to give certain assigment to teacher who asked for it to students. Working in student's goups foment collaboration and solidarity into each person of each team. Solidarity fostered by the teacher when he applied any technic of group, produced a sort of helping among students. Helping is one part of the solidarity of human being. That one is a value that recognice life of another human being y his dignity as a person. The teacher is an agent of change to foster the solidarity through the process teaching - learning, specially when the teacher applies the work in groups in the classroom.

PALABRAS CLAVE: Solidaridad. Valores. Escuela crítica. Maestros.

KEY WORDS: Solidarity. Values. Critical School. Professors.

\section{INTRODUCCIÓN}

El objetivo central del presente estudio es mostrar al profesor como agente de cambio para fomentar la solidaridad en los alumnos a través del proceso de enseñanza aprendizaje. Para este fin, fundamentamos nuestro trabajo en la teoría del modelo pedagógico de la Escuela Crítica elaborada por Margarita Pansza, la cual explicamos en el marco teórico de esta investigación. En el resto del trabajo mostramos las características de ese modelo para saber la manera en la cual los maestros fomentan el valor de la solidaridad y el reconocimiento de la dignidad de las personas a través del proceso de enseñanza aprendizaje fundamentado en aquél paradigma educativo.

\section{MARCO CONCEPTUAL}

Esta investigación se fundamenta en la teoría elaborada por Margarita Pansza acerca de la Escuela Crítica para explicar el papel del profesor como un agente de cambio quién también puede fomentar algunos valores con la aplicación de esta última Escuela en el aula y con su interacción junto a los estudiantes.

La Escuela Crítica es:

Una pedagogía que cuestiona en forma radical tanto los principios de la Escuela Nueva como los de la Escuela Tradicional y la Tecnología Educativa, pronunciándose por la reflexión colectiva entre maestros y alumnos sobre los problemas que los atañen, como un precedente indispensable para la toma de decisiones en el ámbito pedagógico, lo cual implica criticar radicalmente la escuela, sus métodos, sus relaciones, revelando lo que permanece oculto, pero que condiciona su actuación, su forma de ver el mundo, es decir, su ideología (Pansza, M., Pérez E., Morán, P., 2007:57).

La Escuela Crítica es un modelo pedagógico el cual construye espacios democráticos en las aulas para alumnos y maestros. El conocimiento es creado o reconstruído entre estos dos agentes educativos. La manera de construir el conocimiento es en base a la exposición abierta por parte del docente y de los alumnos de un tema o contenido temático perteneciente al programa de estudios. Este contenido se estudiará con la disposición de conocerlo y posteriormente transformarlo. Su transformación es con la finalidad de que beneficie a todos los seres humanos, sobre todo a las personas pobres en economía. 


\section{PLANTEAMIENTO DEL PROBLEMA}

Los profesores son agentes de transformación a través del proceso enseñanza aprendizaje para que los alumnos puedan cambiar los aspectos negativos de la sociedad. El profesor se desenvuelve en un currículo y con la convivencia con otros agentes educativos además que para su ejercicio profesional aplica un modelo pedagógico. La finalidad del currículo es: "favorecer el desarrollo humano y social, el progreso histórico-cultural y la transformación de la escuela" (Lafrancesco, 2004 : 26-27). Con respecto a qué es un modelo pedagógico, María Guadalupe Carranza Peña al referirse a los modelos pedagógicos, menciona que:

Ya desde hace algún tiempo, quizá desde los años sesentas, se viene hablando de modelos, en el ámbito de las ciencias sociales; haciendo alusión a ciertas formas de comportamiento social, económico, educativo, lingüístico, pedagógico, etcétera que se caracterizan, desde mi punto de vista; por dos grandes dimensiones. Una de ellas se refiere a determinada concepción teórico-filosófica que de alguna manera explica aquello que se está tratando de representar en un esquema más o menos simple, pero conformado por supuestos básicos que eventualmente permanecen ocultos, sus principios; y que sin embargo guían la manera de actuar de los sujetos que a ellos se adhieren, supuestos básicos que determinan a la segunda gran dimensión y que se refiere a los aspectos prácticos o procedimentales que dan concreción a tales concepciones.(Carranza, 2004 : 213).

Un modelo es una explicación de la realidad. Brian Wilson argumenta el concepto de modelo de la siguiente manera:
Un modelo es la interpretación explícita de lo que uno entiende de una situación, o tan sólo de las ideas de uno acerca de esa situación. Puede expresarse en matemáticas, símbolos o palabras, pero en esencia es una descripción de entidades, procesos o atributos y las relaciones entre ellos. Puede ser prescriptivo o ilustrativo, pero, sobre todo, debe ser útil (Wilson, 1993: 28).

La Escuela Crítica es un modelo pedagógico. Ante los anteriores argumentos podemos elaborar la siguientes preguntas de investigación: ¿Cuáles son las características de la Escuela Crítica que permiten el fomento de la solidaridad en el proceso de enseñanza aprendizaje? ¿Con cuál técnica de enseñanza el maestro fomenta la solidaridad a través del proceso de enseñanza aprendizaje?

\section{MÉTODOS}

El método empleado en la presente investigación fue el método deductivo porque a partir de un todo general como lo es la teoría de la Fundamentación y Operatividad de la didáctica de Margarita Pansza et al., se toman ciertos postulados para aplicarlos a este estudio.

\section{LA FUNCIÓN DEL PROFESOR SEGÚN EL MODELO DE LA ESCUELA CRÍTICA}

El maestro es un agente de cambio que puede fomentar la solidaridad entre los estudiantes. Esto se lleva a cabo mediante la implementación de la Escuela Crítica. El significado de ésta la mencionamos en el marco teórico del presente trabajo. Una característica de la Escuela Crítica es la idea y actividad de transformar el conocimiento. Esto último significa la modificación del saber tradicional. La transformación del objeto de estudio la realizan alumnos y docentes. 
Debido a que profesor y alumnos estudian juntos un contenido temático y analizan temas, posteriormente surguen entre los agentes de cambio propuestas las cuales mejoran a las instituciones, conceptos, valores y saberes.

Cualquier institución, concepto, valor o saber creado por las personas se puede transformar para mejorar la calidad de vida. Pansza menciona que las instituciones se pueden transformar. "Las instituciones son algo que crean los hombres $y$ en el poder colectivo que crean está el germen de su propia transformación" (Pansza et al., 2007: 58).

Un gran mérito de la Escuela Crítica es llevar a cabo la transformación del conocimiento. Docentes y alumnos realizan este cambio en el salón de clases sin un escritorio, con mesas agrupadas en semicírculo y con una actitud abierta para modificar conceptos, valores e instituciones y estos puedan propiciar mejores condiciones de vida de las personas. Esta transformación del conocimiento realizada por los agentes educativos antes mencionados y de la manera antes mencionada, nos indica que éstos llevan acabo el cambio con el currículo oculto de la institución educativa.

Otra característica de la Escuela Crítica es la construcción del conocimiento en grupos. En este modelo pedagógico, los estudiantes trabajan en equipos dentro y/o fuera del salón de clases. Cuando se lleva a cabo lo anterior, los integrantes del equipo se retroalimentan para entregar un trabajo solicitado por el maestro.

La conformación de equipos es muy variada. Existen diversas técnicas de enseñanza de grupos. En la actualidad podemos encontrar una gran bibliografía relativa a estas últimas.

El trabajo en equipo de alumnos fomenta a la solidaridad de cada persona para el ente colectivo el cual es el equipo. El docente es un potencial agente de cambio para fomentar la solidaridad a través del proceso de enseñanza aprendizaje, en especial cuando éste aplica el trabajo en equipo en el aula.

Uno de los motivos por los cuales la Escuela Crítica hacer trabajar a los alumnos en equipo es para desarrollar y fomentar la solidaridad en las personas. Esta última sirve a los alumnos para su futuro profesional y es de utilidad a los egresados que se integran al mercado de trabajo para que puedan corregir los defectos que hay en este último.

La Escuela Crítica fomenta la participación de todos los alumnos integrantes de una clase por diversos motivos. El primero y más importante es para que todos los alumnos desarrollen el hábito de argumentar y exponer sus ideas. Otro motivo menos importante es para que se cumpla el principio de la Escuela Crítica que postula a la educación como verdadero espacio democrático.

Los profesores que aplican los postulados de la Escuela Crítica, fomentan valores en los alumnos. Entre estos se encuentra la solidaridad. Unos valores que se necesitan ejercer en la actualidad son ésta y la honradez. Estos últimos pueden ayudar a combatir la corrupción. A mayor implementación de la Escuela Crítica por parte de los maestros, más alumnos educados para actuar con solidaridad. A mayor implementación de la Escuela Crítica por parte de los docentes, mayor número de alumnos egresados educados para actuar con solidaridad. Esta última ayuda a reconocer la dignidad de las personas.

Todas las personas tenemos dignidad. Emmanuel Kant argumentó acerca de la esta última lo siguiente: "aquello que constituye la condición para que algo sea fin para sí mismo, eso no tiene meramente valor relativo o precio, sino un valor interno, esto es dignidad" (Kant, 2013: 53). La solidaridad - fomentada en la Escuela Crítica -es una acción con valor moral para alcanzar la dignidad. La honradez es otra 
acción moral para alcanzar la dignidad. La corrupción no es una acción ni un valor moral, por lo que no forma parte de la dignidad de las personas. Por lo tanto, la corrupción debe ser combatida y eliminada.

Kant argumentó que todas las personas somos fines en sí mismos, no simples medios para los fines de otras personas.

La Escuela Crítica fomenta a la solidaridad en los alumnos en el momento en el cual el docente aplica cualquier técnica de enseñanza en equipos - por ejemplo panel, casos prácticos o cualquier otra dinámica de grupos.

La solidaridad fomentada por el docente cuando aplica cualquier técnica grupal, produce una especie de ayuda entre los estudiantes. La ayuda es una parte de la solidaridad del ser humano. La primera es un valor que permite reconocer la vida de otro ser humano y su dignidad como persona. En la virtud de la amistad se encuentra a la ayuda como una especie de aquélla. La Escuela Crítica fomenta a la ayuda porque ésta se encuentra en una parte de la solidaridad y como sostuvimos en párrafos anteriores, esta última se presenta cuando el docente aplica cualquier técnica de enseñanza en equipos. Ronald Dworkin argumenta la siguiente idea de la ayuda:

La riqueza y la suerte se reparten de manera muy despareja entre los seres humanos, de modo que a menudo nos encontramos en la situación de ayudar a extraños que están peor que nosotros, sea en general o porque han sufrido algún accidente o corren algún peligro en particular (Dworkin, 2014: 335).

La persona que ayuda a otro ser humano reconoce la dignidad que le pertenece a la humanidad. Dworkin escribió acerca de esta idea: "Debemos mostrar pleno respeto por la igual importancia objetiva de la vida de todas las personas, pero también pleno respeto por nuestra responsabilidad de hacer algo valioso de nuestra vida propia" (Dworkin, 2014: 335).

La Escuela Crítica fomenta valores y el respeto por la dignidad de todos los agentes educativos. En este modelo pedagógico el maestro no emplea el estrado del aula y en cambio trabaja en el mismo nivel físico de los alumnos. A su vez estos últimos reconocen al docente como a una persona con más conocimientos que los estudiantes. En este acto el profesor respeta a la dignidad de los alumnos cuando trabaja hombro a hombro con estos últimos porque no emplea el estrado y a su vez los estudiantes respetan a la dignidad del docente porque reconocen todo el saber que el maestro ha acumulado a lo largo de los años.

El profesor es un agente de cambio para fomentar la solidaridad en el aula a través del trabajo en grupos y de su trabajo en el aula sin estrado. Muchas instituciones cuentan con un estrado en sus salones de clase. Es recomendable que se elimine aquél para que se que no exista la separación entre profesores y alumnos con el fin de que estos últimos sigan con la práctica de ciertos valores como el de la solidaridad.

\section{CONCLUSIONES}

A) La Escuela Crítica es un modelo pedagógico el cual construye espacios democráticos en las aulas para alumnos y maestros. El conocimiento es creado o reconstruído o transformado entre estos dos agentes educativos.

B) Docentes y alumnos realizan actividades en el salón de clases sin un escritorio, con mesas agrupadas en semicírculo y con una actitud abierta para modificar conceptos, valores e instituciones y estos puedan propiciar mejores condiciones de vida de las personas. Esta transformación del conocimiento realizada por los agentes educativos antes mencionados y de la manera antes mencionada, nos indica 
que éstos llevan acabo el cambio en el currículo oculto de la institución educativa.

C) El trabajo en equipo de alumnos fomenta la colaboración y la solidaridad de cada persona para el ente colectivo el cual es el equipo. El docente es un potencial agente de cambio para fomentar la solidaridad a través del proceso de enseñanza aprendizaje, en especial cuando éste aplica el trabajo en equipo en el aula.

D) La solidaridad fomentada por la Escuela Crítica, al aplicar el docente cualquier técnica grupal, produce una especie de ayuda entre los estudiantes. La persona que ayuda a otro ser humano reconoce la dignidad que le pertenece a la humanidad. La dignidad significa que todas las personas somos fines y no medios otras personas.

\section{REFERENCIAS BIBLIOGRÁFICAS}

Carranza Peña, M. (2004). De la Didáctica Tradicional al Constructivismo. En Anzaldúa Arce, Raúl y Ramírez Grajeda, Beatriz, (coordinadores), Formación y tendencias educativas (p. 213). México: Universidad Autónoma Metropolitana Unidad Azcapotzalco.

Dworkin, R. (2014). Justicia para erizos. México: Editorial Fondo de Cultura Económica.

Kant, M. (2013). Fundamentción Metafísica de las costumbres. México: Editorial Porrúa.

Lafrancesco V., G. (2004). Currículo y Plan de estudios. Estructura y Planeamiento. Bogotá: Cooperativa Editorial Magisterio.

Pansza, M., Pérez, C. Morán, P. (2007). Fundamentación de la didáctica. México: Editorial Gernika.

Wilson, B. (1993). Sistemas: conceptos, metodología y aplicaciones, México: Editorial Limusa. 